\title{
炉底漏风对炉内燃烧及 $\mathrm{NO}_{x}$ 生成排放特性的影响*
}

\author{
刘铭媛陈国庆 戴维葆 孙俊威 \\ (清洁高效燃煤发电与污染物控制国家重点实验室(国电科学技术研究院有限公司) 南京 210023)
}

\begin{abstract}
摘要: 干式排渣系统的冷却风会影响炉内燃烧的组织, 现有研究多采用现场试验的方法, 研究对象多为排烟温度或锅炉效率。 以一台湿渣机改干渣机的 $660 \mathrm{MW}$ 超临界机组为研究对象, 采用数值模拟的方法, 研究了干渣机漏风对炉内燃烧和 $\mathrm{NO}_{x}$ 生成 排放特性的影响, 对比分析了不同炉底漏风来源时, 炉膛火焰中心、飞灰含碳量、 $\mathrm{NO}_{x}$ 排放浓度等关键参数的变化。研究结 果表明, 炉底漏风量为燃尽风减量的工况对于炉内燃烧影响不明显; 炉底漏风量全部或部分为燃烧器二次风减量时, 随着漏 风量占二次风比例的增大, 主燃区氧量水平、出口飞灰含碳量、 $\mathrm{NO}_{x}$ 排放浓度均随之增高。从冷却风量对炉内燃烧及 $\mathrm{NO}_{x}$ 排 放特性的角度分析了干渣机不同冷却风来源对机组运行的影响, 为干式排渣机设计、改造及运行提供理论指导。
\end{abstract}

关键词: 煤粉燃烧; 炉底漏风; 干式排渣; 数值模拟

中图分类号: TK227

\section{Influence of Air Leakage on the $\mathrm{NO}_{x}$ Emission and Combustion Characteristics}

\author{
LIU Mingyuan CHEN Guoqing DAI Weibao SUN Junwei \\ (State Key Laboratory of Clean and Efficient Coal-fired Power Generation and Pollution Control \\ (GUODIAN Science and Technology Research Institute), Nanjing 210023)
}

\begin{abstract}
The cooling wind of the dry slagging system will affect the burning organization in the furnace. The existing research mostly adopts the field test method, and the research object is mostly the exhaust gas temperature or the boiler efficiency. Taking a $660 \mathrm{MW}$ supercritical unit of a wet slag machine to dry slag machine as the research object, the influence of air leakage from dry slag machine on combustion and $\mathrm{NO}_{x}$ emission characteristics of the furnace was studied by numerical simulation method. At the source, changes in key parameters such as furnace flame center, fly ash carbon content, and $\mathrm{NO}_{x}$ emission concentration. The results show that the air leakage at the bottom of the furnace is the same as that of the burned-out wind. The influence of the air leakage at the bottom of the furnace is not obvious. When the air leakage at the bottom of the furnace is all or part of the secondary air reduction of the burner, the air leakage accounts for the secondary air. As the proportion increases, the oxygen level in the main combustion zone, the carbon content of the outlet fly ash, and the $\mathrm{NO}_{x}$ emission concentration increase accordingly. From the perspective of the cooling air volume on the combustion and $\mathrm{NO}_{x}$ emission characteristics of the furnace, the influence of different cooling air sources of the dry slag machine on the unit operation is analyzed, which provides theoretical guidance for the design, modification and operation of the dry slag discharge machine.
\end{abstract}

Key words: pulverized coal combustion; bottom leakage; dry slag discharge; numerical simulation

\section{0 前言}

干式排渣技术具有节能、节水、环保和综合效 益高等优点, 成为水资源贵乏地区火电机组首选冷 渣技术, 在国内外燃煤发电机组得到了广泛的应用。

\footnotetext{
* 中国国电集团公司资助项目(D2017Y006)。20180914 收到初稿, 20190815 收到修改稿
}

该技术通常采用环境空气作为炉渣和干渣机的冷却 介质。进入干渣机的冷却风量不可控, 主要取决于 炉膛负压。新建机组锅炉燃烧系统设计时通常会考 虑干渣机的冷却风量, 但由于干渣机的冷却风量是 根据锅炉最大出力设计, 由此导致中低负荷下干渣 机的漏风率远高于设计值。对于湿式排渣系统改干 式排渣系统的机组, 由于锅炉最初的炉底漏风是依 据湿式排渣系统设计, 取值较低, 改为干式排渣系 统后炉底漏风高的问题不仅出现于中低负荷下, 高 
负荷下也非常突出。从干渣机漏入炉膛的风为无组 织漏风, 大量无组织漏风进入炉内会影响燃烧的组 织, 导致炉膛火焰中心偏移、过热器和再热器超温 严重、减温水量大、排烟温度升高、机组运行经济 性降低等问题。因此, 干渣机漏风问题得到广泛的 关注。

针对干渣机漏风对机组运行和性能的影响, 国 内研究者开展了相关研究。高继录等 ${ }^{[3]}$ 采用现场试 验方法, 分析了干式排渣系统冷却风比例、钢带转 速对锅炉效率的影响, 并对比了额定负荷和中低负 荷下干式和湿式排渣系统, 给出了干式排渣系统对 锅炉效率影响的幅度。范仁东 ${ }^{[4]}$ 基于 4 家电厂的试 验数据分析了风冷干排渣系统对锅炉效率及运行经 济性的影响, 对比了风冷干式和水冷湿式排渣系统, 认为干排渣系统是一种难以满足节能减排政策的设 备。王建勋等 ${ }^{[5]}$ 针对 $\mathrm{W}$ 型火焰锅炉进行了炉底漏风 试验研究, 对比了排渣机关断门和冷却风门全开和 全关 2 个各工况下锅炉排烟温度的变化, 给出了炉 底漏风对机组经济性的影响。夏文静等 ${ }^{[6]}$ 采用在线 风量测试方法对排渣系统进行了漏风量测试, 并分 析了漏风对排烟温度、锅炉效率及省煤器出口烟温 的影响。许华等 ${ }^{[7]}$ 测试了某 $1000 \mathrm{MW}$ 锅炉干式排 渣系统的运行数据, 建立了干式排渣系统冷却风计 算模型、湿式排渣系统过冷沸腾蒸汽量计算模型, 及送风温度偏差和给水温度偏差对排烟温度修正模 型, 对比了干湿式排渣系统对锅炉效率的影响。

综上可知, 关于干式排渣系统冷却风对机组 运行影响的研究多采用现场试验的方法, 研究对 象多为排烟温度或锅炉效率, 针对不同冷却风来 源对炉内燃烧及 $\mathrm{NO}_{x}$ 排放特性的研究鲜见报道。 本文以一台湿渣机改干渣机的 $660 \mathrm{MW}$ 机组为研 究对象, 采用数值模拟的方法对有、无漏风以及 不同漏风来源下炉内燃烧过程和 $\mathrm{NO}_{x}$ 排放特性进 行了数值模拟研究, 对比分析了各工况下炉膛火 焰中心、飞灰含碳量、 $\mathrm{NO}_{x}$ 排放浓度等参数的变 化, 从另一个角度详细地分析了干渣机冷却风对 机组运行的影响, 为干式排渣机设计、改造及运 行提供理论指导。

\section{1 研究对象}

本文研究对象为某电厂 DG2141/25.4-II7 型锅 炉。该锅炉为超临界参数直流炉, 采用一次中间再 热、单炉膛、平衡通风、尾部双烟道。炉膛断面尺 寸为 $22.16 \mathrm{~m}$ 宽、 $15.46 \mathrm{~m}$ 深。制粉系统为正压直吹 式, 配备 6 台中速磨煤机。燃烧器为 DBC-OPPC 型
低 $\mathrm{NO}_{x}$ 燃烧器, 采用前后墙对冲布置, 共 36 只, 前墙 18 只, 后墙 18 只, 各分 3 层布置, 每层 6 只。 分别在前后墙距离最上层燃烧器一定距离处布置了 一层燃尽风(Over fire air, OFA)喷口, 每层 8 只, 其 中, 2 只侧燃尽风喷口位置略低于 6 只燃尽风喷口。 每层风室入口处均设置二次风挡板用以调整风室的 进风量, 二次风风门挡板由气动执行器进行调节。 炉膛四周为全焊膜式水冷壁, 由下部螺旋盘绕上升 水冷壁和上部垂直上升水冷壁 2 种结构组成。

\section{2 数学模型及计算方法}

\section{1 网格划分}

根据燃烧器与炉膛实际物理结构的尺寸及特点 建立了物理模型, 为了更加精细地划分网格, 本文 选取了一半锅炉结构作为数值模拟对象, 如图 1 所 示。将炉膛划分为 4 个区域, 分别为冷灰斗区、燃 烧器区、燃尽风区、屏式过热器区, 为减少网格数 目, 炉膛上部区域采用了结构化网格, 同时屏式受 热区以简化后的过热屏为基准进行了规整分区并划 分六面体结构化网格, 冷灰斗区域则采用了非结构 化网格。整个计算域在尽可能顺应流场流向的础上, 划分六面体结构化网格, 以减少网格数目, 从而提 高计算速度。对网格进行了独立性分析后, 最终整 个炉膛网格总数约为 700 万左右。

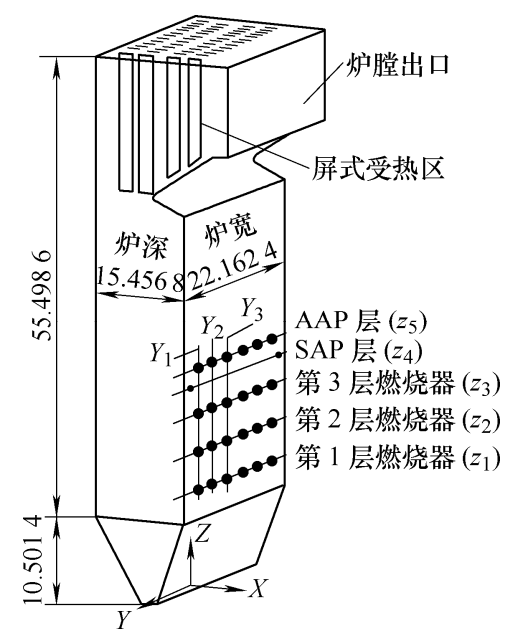

图 1 炉膛结构简图

\section{2 数学模型}

针对前后墙对冲燃煤锅炉特点, 经查阅相关文 献 ${ }^{[8-12]}$ 及试算, 确定数值模拟采用三维稳态计算, 采用带旋流修正的 Realizable k- $\varepsilon$ 模型计算气相湍流 流动, 采用颗粒随机轨道模型模拟煤粉颗粒的运动, 采用双平行竞争反应模型模拟挥发分的析出, 采用 混合分数-概率密度函数模型模拟气相湍流燃烧, 采 用动力/扩散控制反应速率模型模拟焦炭的燃烧, 采 
用 P-1 辐射模型计算炉内辐射换热。采用半隐格式 压力关联方程(SIMPLE)算法求解离散方程组的压 力和速度的耦合, 并结合标准壁面函数将壁面上的 物理量与炉内湍流核心区的物理量联系起来对壁面 区的流场进行计算。

\section{3 计算方法及条件}

炉内燃烧过程计算分两步进行: 首先, 进行单 只燃烧器内的气固流场模拟, 然后, 通过 UDF 编译 程序将单只燃烧器喷口条件计算结果作为炉膛计算 的入口边界条件, 使得模拟更贴近实际运行中真实 的两相流程分布形态, 计算结果更加精确。对燃烧 器的模拟计算采用组分运输模型和 DPM 模型求解, 使连续相与颗粒相之间相互耦合。由于炉膛数值模 拟具有强非线性特点, 在计算迭代时, 先进行冷态 工况计算, 获得残差曲线收玫稳定的空气流场, 再 以此作为初始条件进行热态计算, 直至收玫。 $\mathrm{NO}_{x}$ 的计算采用后处理的方法, 且只考虑热力型 $\mathrm{NO}_{x}$ 和 燃料型 $\mathrm{NO}_{x}$ 。飞灰含碳量的计算采用碳平衡法。

本文对干渣机漏风的来源分为三种情况开展计 算。(1) 干渣机漏风量为燃烧器二次风的减量; (2) 干 渣机漏风量为燃尽风的减量; (3) 干渣机漏风量为燃 烧器二次风的减量与燃尽风的减量之和。定义炉底 漏风率为炉底漏风量占二次风量的百分比, 共开展 了 4 个工况的计算; (1) $0 \%$, 基础工况, 炉底完全无 漏风, 即干渣机改造前湿渣机运行工况; (2) 6\%RSQ 工况, 炉底漏风量占二次风量的 $6 \%$, 漏风量为 燃烧器二次风的减量; (3) 6\%OFA 工况, 炉底漏 风量占二次风风量的 6\%, 漏风量为燃尽风的减量; (4) 3\%RSQ3\%OFA 工况, 炉底漏风量占二次风量的 $6 \%$, 其中漏风量的 $50 \%$ 取自燃烧器二次风, $50 \%$ 取自燃尽风。4 个工况模拟均在 BRL工况下进行, 后墙第三层燃烧器处于备用状态, 燃用煤量及总风 量保持不变。

\section{3 结果与分析}

\section{1 炉内燃烧特性的变化}

\subsection{1 炉内温度场}

图 2 为不同工况下炉膛中心纵截面温度场分 布。比较图中各工况下炉膛内大于 $1800 \mathrm{~K}$ 的高温区 可知, 炉底无漏风工况下, 高温区主要集中在第三 层燃烧器附近; 其他炉底漏风的工况下, 高温区覆 盖在燃尽风层附近的区域, 很明显炉内最高温度的 位置上移, 即炉膛火焰中心上移。这与实际运行过 程中炉底漏风增大, 过热器超温, 减温水量增大的 现象相符 ${ }^{[15]}$ 。

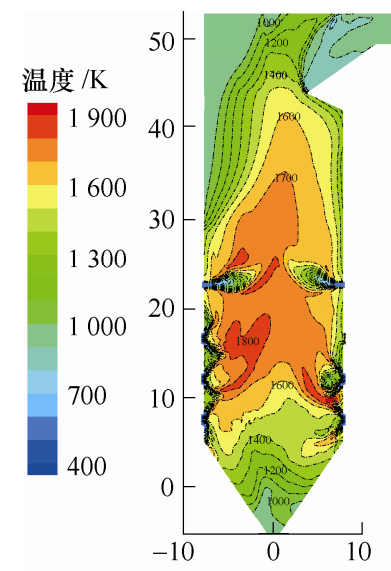

(a) $0 \%$

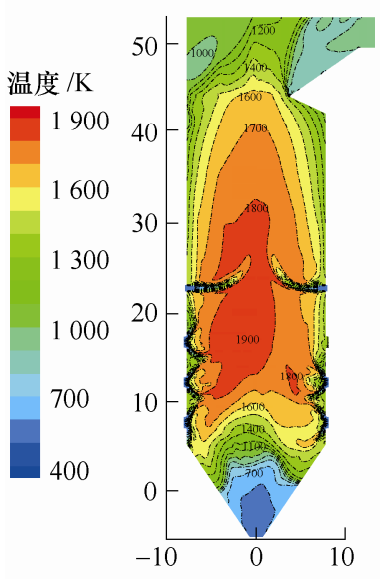

(c) $6 \% \mathrm{OFA}$

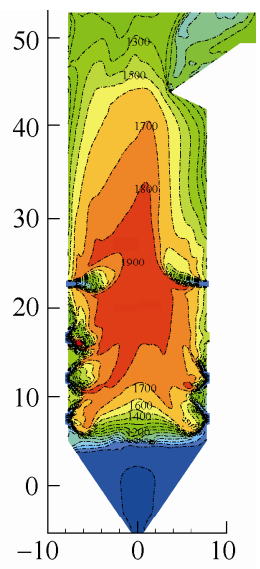

(b) $6 \% \mathrm{RSQ}$

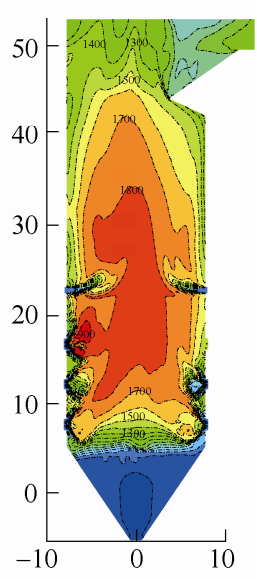

(d) $3 \%$ RSQ $3 \%$ OFA
图 2 炉膛中心截面的温度场分布

另外, 由图 2 中可以看出, 炉底漏风对炉膛下部 冷灰斗区域烟气温度影响显著。漏风率为 $0 \%$ 时, 炉 膛下部区域烟气温度相对较高, 均高于 $900 \mathrm{~K}$; 其他 炉底漏风的工况下, 冷灰斗区域温度均有不同程度的 降低，在 $400 \sim 700 \mathrm{~K}$ 之间，且低温区向上影响至主 燃区, 第 1 层燃烧器喷口区域的烟气温度受到炉底漏 风影响, 降到了 $1500 \mathrm{~K}$ 以下; 燃烧器喷口温度降低 会导致煤粉着火延迟, 不利于煤粉的燃尽。

\section{1 .2 炉内氧量分布}

图 3 给出了不同工况下炉膛中心纵截面 $\mathrm{O}_{2}$ 浓度 的分布情况。由图可知, 炉底无漏风工况下, 由于 采用空气分级燃烧技术, 主燃区处于缺氧条件下运 行, 因此, 炉膛中心氧量很快被消耗殆尽, 炉膛中 心区域氧量基本为零。当冷风由炉底进入, 炉膛下 部冷灰斗低温区氧浓度升高, 且富氧区向上部主燃 区扩展。对比分析炉底漏风的 3 种工况可知, 炉底 漏风量全部为燃尽风减少量 $(6 \% \mathrm{OFA})$ 工况下，主燃 区上部氧浓度小于 0.01 ; 漏风量的 $50 \%$ 取自燃烧器 二次风、 $50 \%$ 取自然尽风( $3 \% \mathrm{RSQ} 3 \% \mathrm{OFA})$ 工况下, 主燃区氧浓度在 $0.01 \sim 0.05$ 之间; 炉底漏风量全部 为燃烧器二次风减少量(6\%RSQ)工况下, 主燃区氧 
浓度在 $0.02 \sim 0.08$ 之间。可见, 若炉底漏风量全部 为燃烧器二次风的减少量, 则对于主燃区氧浓度的 影响会较大, 且主燃区氧浓度随着漏风量占燃烧器 二次风量比例的增大而增大。这是由于主燃区二次 风量减少, 使得燃烧器的旋流强度减弱, 喷口回流 区减小, 卷吸高温烟气的能力减弱, 因此, 煤粉的 着火延迟, 在主燃区燃尽变差, 导致主燃区的运行 氧浓度水平显著升高。

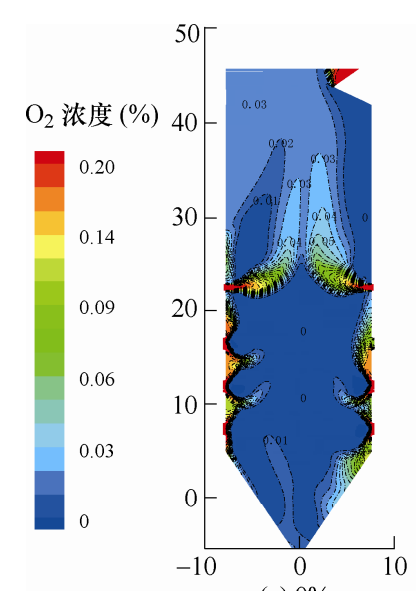

(a) $0 \%$

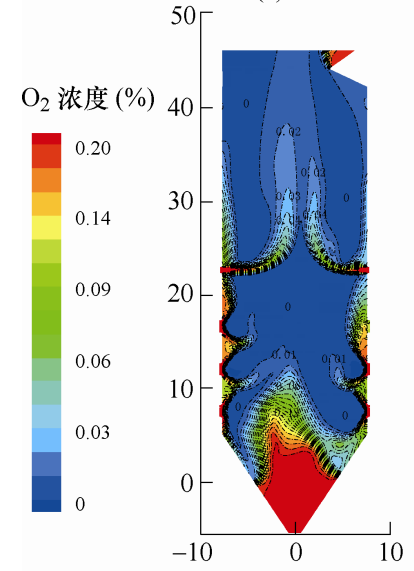

(c) $6 \% \mathrm{OFA}$

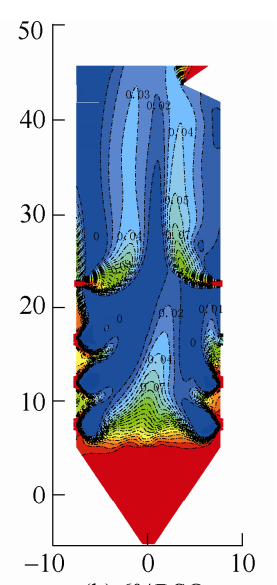

(b) $6 \% \mathrm{RSQ}$

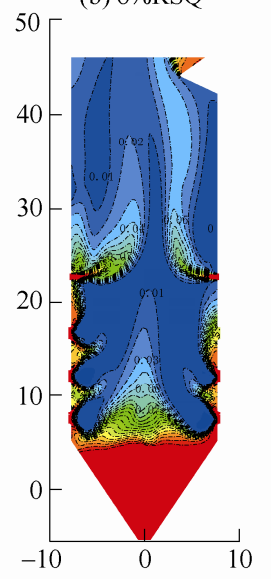

(d) $3 \% \mathrm{RSQ} 3 \% \mathrm{OFA}$
图 3 炉膛中心截面 $\mathrm{O}_{2}$ 浓度分布

\subsection{3 飞灰含碳量}

飞灰含碳量可以表征煤粉的燃尽程度, 是衡量 燃烧效果的重要参数。采用碳平衡计算方法, 计算 得到了各工况下炉膛出口截面上飞灰含碳量的平均 值, 计算结果见图 4。

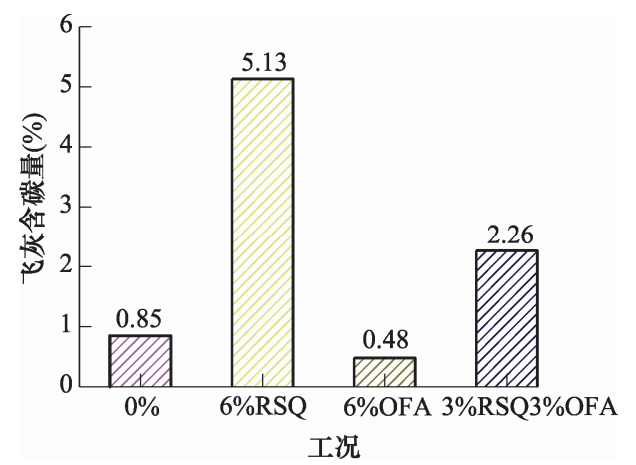

图 4 漏风对飞灰含碳量的影响
由图中可以看出, 炉底漏风量全部为燃尽风减 少量 $(6 \% \mathrm{OFA})$ 的工况下飞灰含碳量最低, 甚至低于 炉底无漏风的工况, 这是由于由炉底的漏风一定程 度上对主燃区的燃烧补给了氧量, 使煤粉燃烧更充 分, 从而降低了烟气中的飞灰含碳量。

炉底漏风量全部为燃烧器二次风减少量 (6\%RSQ)的工况下飞灰含碳量最高, 达到了 $5.13 \%$, 这是由于主燃区二次风量减少, 使燃烧器喷口回流 区缩小, 同时, 在炉底冷风进入主燃区的因素叠加 作用下, 燃烧器喷口附近温度水平降低, 导致煤粉 着火推迟、燃尽差, 此结果印证了图 3b 中氧量浓度 水平为各工况下最高的计算结论。

从图中还可以看出, 有炉底漏风的工况下, 飞 灰含碳量随着漏风量占燃烧器二次风量的比例增大 而增大, 这与图 3 炉膛氧量浓度场的计算结论是一 致的。漏风量占燃烧器二次风量的大小对煤粉燃尽 有着直接的影响。

\section{$3.2 \mathrm{NO}_{x}$ 排放特性的变化}

图 5 给出了不同工况下炉膛中心纵截面 $\mathrm{NO}_{x}$ 浓 度的分布情况。由图可知, 炉底无漏风时, 主燃区

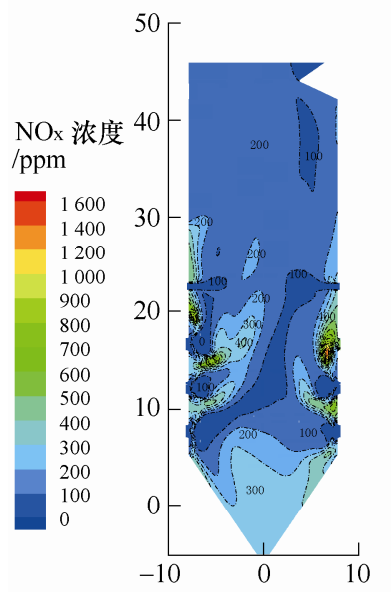

(a) $0 \%$

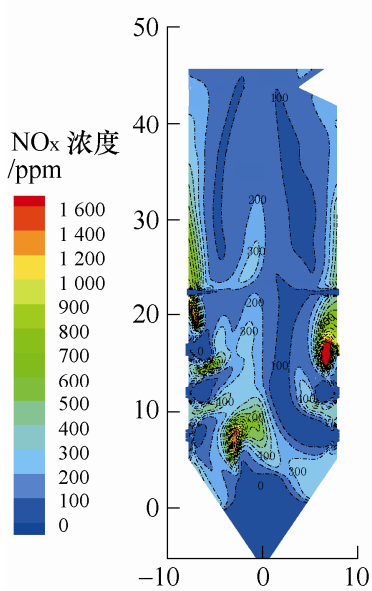

(c) $6 \% \mathrm{OFA}$

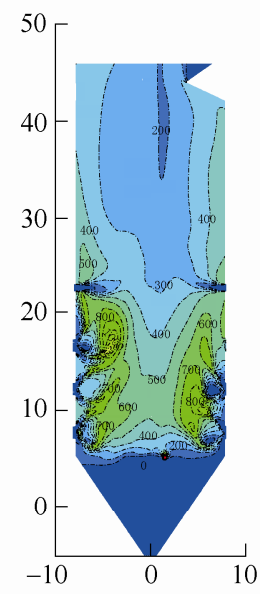

(b) $6 \% \mathrm{RSQ}$

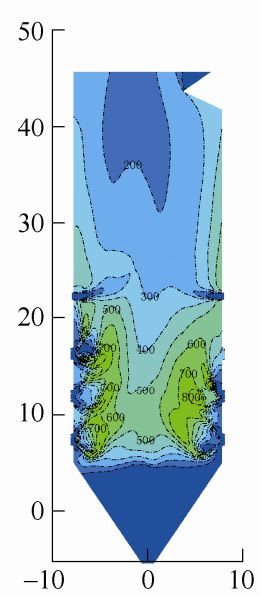

(d) $3 \% \mathrm{RSQ} 3 \% \mathrm{OFA}$
图 5 炉膛中心截面 $\mathrm{NO}_{x}$ 浓度分布 
产生的 $\mathrm{NO}_{x}$ 浓度最低, 只有 $100 \sim 200 \mathrm{ppm}$ 。其他 3 种工况由于炉底冷风的进入, 使炉内主燃区运行氧 量升高, 在高温、氧化气氛下, 热力型及燃料型 $\mathrm{NO}_{x}$ 大量产生, 从而主燃区的 $\mathrm{NO}_{x}$ 浓度均有所增大。

分析炉底漏风的 3 种工况下主燃区产生的 $\mathrm{NO}_{x}$ 浓度场可知, 相比于其他两种工况, 炉底漏风量全 部为燃尽风减少量 $(6 \% \mathrm{OFA})$ 工况下，主燃区 $\mathrm{NO}_{x}$ 浓 度最低, 仅略高于无漏风工况, 约为 $200 \mathrm{ppm}$ 左右; 漏风量的 $50 \%$ 取自燃烧器二次风、50\%取自燃尽风 (3\%RSQ3\%OFA)工况, 与炉底漏风量全部为燃尽风 减少量 $(6 \% \mathrm{RSQ})$ 工况下, 主燃区 $\mathrm{NO}_{x}$ 浓度都较高, 均达到了 $500 \mathrm{ppm}$ 左右, 且这两种工况下 $\mathrm{NO}_{x}$ 分布 规律较为相似。比较图 3 可知, 主燃区 $\mathrm{NO}_{x}$ 浓度分 布规律与主燃区氧浓度分布规律相对应, 即 $\mathrm{NO}_{x}$ 浓度随漏风量占燃烧器二次风量比例的增大而升 高。这是由于炉底漏风改变了炉膛内部氧量场的分 布, 主燃区二次风量减少使炉内燃烧组织变差, 氧 化环境下 $\mathrm{NO}_{x}$ 大量生成, 导致空气分级燃烧的效 果不佳。

分别计算得不同工况时炉膛出口 $\mathrm{NO}_{x}$ 浓度平均 值并绘于图 6 中, 可以看出, 基础工况的炉膛出口 $\mathrm{NO}_{x}$ 浓度为 $415 \mathrm{mg} / \mathrm{Nm}^{3}\left(@ 6 \% \mathrm{O}_{2}\right)$, 是所有计算工 况下的最低值。可见, 炉底漏风会提高锅炉 $\mathrm{NO}_{x}$ 的 排放水平。

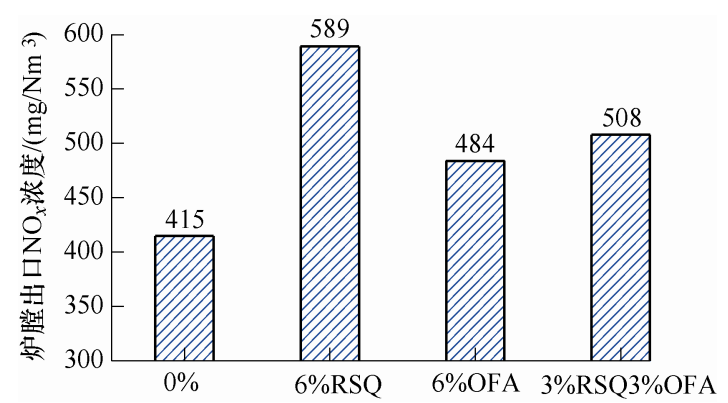

图 6 漏风对炉膛出口 $\mathrm{NO}_{x}$ 浓度的影响

比较三个炉底有漏风工况, 可以看出, 炉底漏 风量全部为燃尽风减少量 $(6 \% \mathrm{OFA})$ 工况下, 炉膛出 口 $\mathrm{NO}_{x}$ 浓度较低, 为 $484 \mathrm{mg} / \mathrm{Nm}^{3}\left(@ 6 \% \mathrm{O}_{2}\right)$; 其次 为漏风量的 $50 \%$ 取自燃烧器二次风、 $50 \%$ 取自燃尽 风( $3 \% \mathrm{RSQ} 3 \% \mathrm{OFA})$ 工况, 这时 $\mathrm{NO}_{x}$ 排放浓度为 508 $\mathrm{mg} / \mathrm{Nm}^{3}\left(@ 6 \% \mathrm{O}_{2}\right)$; 炉底漏风量全部为燃烧器二次 风减少量 $(6 \% \mathrm{RSQ})$ 工况的炉膛出口 $\mathrm{NO}_{x}$ 浓度最高, 达到了 $589 \mathrm{mg} / \mathrm{Nm}^{3}\left(@ 6 \% \mathrm{O}_{2}\right)$ 。此计算结果与图 5 炉膛 $\mathrm{NO}_{x}$ 浓度场的计算结果一致, 炉膛出口 $\mathrm{NO}_{x}$ 浓度随着炉底漏风量占燃烧器二次风量比例的增大 而升高。结合炉底漏风对炉膛氧量水平、飞灰含碳 量、 $\mathrm{NO}_{x}$ 浓度场的计算分析可以得出, 干渣机引起 的炉底漏风会改变炉内氧量场、 $\mathrm{NO}_{x}$ 浓度场的分布,
炉底漏风量占燃烧器二次风量的比例大小影响炉内 燃烧状况，这是由于主燃区二次风量减少，使得燃 烧器旋流强度减弱, 从而卷吸高温烟气的能力减弱, 导致主燃区的燃烧组织变差, 煤粉着火推迟、不易 燃尽, 炉膛氧量水平升高, 飞灰含碳量升高。炉膛 高温、富氧的气氛使热力型及燃料型 $\mathrm{NO}_{x}$ 产生量增 多, 炉膛出口 $\mathrm{NO}_{x}$ 排放浓度升高。此现象随着炉底 漏风量占燃烧器二次风量的比例增大而更加明显, 炉底漏风量占燃烧器二次风的比例越大, 炉膛氧量 水平越高、 $\mathrm{NO}_{x}$ 浓度越大、出口飞灰含碳量越高。

\section{4 数值模拟结果与实测结果的对比}

为验证模拟结果的准确性, 对研究对象本体在 BRL 工况实际运行中炉膛出口 $\mathrm{NO}_{x}$ 进行检测, 并将 检测结果与模拟计算的炉膛出口 $\mathrm{NO}_{x}$ 浓度进行对比 见表 1 。

表 1 模拟结果与实测结果对比

\begin{tabular}{cccc}
\hline 工况 & $\begin{array}{c}\text { 实测结果/ } \\
\left(\mathrm{mg} / \mathrm{Nm}^{3}\right)\end{array}$ & $\begin{array}{c}\text { 模拟结果/ } \\
\left(\mathrm{mg} / \mathrm{Nm}^{3}\right)\end{array}$ & $\begin{array}{c}\text { 偏差 } \\
(\%)\end{array}$ \\
\hline $6 \% \mathrm{OFA}$ & & 484 & 9.2 \\
$3 \% \mathrm{RSQ3} \% \mathrm{OFA}$ & 533 & 508 & 4.7 \\
$6 \% \mathrm{OFA}$ & & 589 & 10.5 \\
\hline
\end{tabular}

由表 1 模拟结果与实测结果对比可知, $3 \% \mathrm{RSQ} 3 \% \mathrm{OFA}$ 工况与实际运行条件较为相近, 其 模拟结果与实测结果偏差为 $4.7 \%$, 证明模拟计算结 果较为准确。

\section{5 结论}

采用数值模拟方法, 对不同漏风来源对于炉内 燃烧过程和 $\mathrm{NO}_{x}$ 排放的影响进行了研究, 得到以下 结论。

(1)由于燃尽风量的减少对于炉内燃烧产生的 影响较小, 故而炉底漏风量为燃尽风减量的工况对 于炉内燃烧影响不明显, 但使炉膛出口 $\mathrm{NO}_{x}$ 排放浓 度略有增高。

(2)由于主燃区二次风量减少会导致燃烧器旋 流强度减弱, 煤粉着火延迟、燃尽差, 因此, 炉底 漏风量全部或部分为燃烧器二次风减量时, 随着漏 风量占二次风比例的增大, 主燃区氧量水平、出口 飞灰含碳量、 $\mathrm{NO}_{x}$ 排放浓度均随之增高。

(3)炉底漏风为燃尽风减少量相对于燃烧器二 次风减少量对炉内燃烧影响更小，因此，采用干渣 机的机组实际运行过程中在总风量一定情况下应优 先保证足够的主燃区二次风量, 在 $\mathrm{NO}_{x}$ 排放可控的 
前提下, 适当减少燃尽风量。

\section{参 考 文 献}

[1] 于长友. 燃煤电厂钢带式排渣机干式除渣技术 [J]. 中 国电力, 2007, 40(1): 56-59.

YU Changyou. Dry bottom ash air-cooling conveyor handling system in coal-fired power plants[J]. Electric Power, 2007, 40(1): 56-59.

[2] 柳晓, 刘振强, 陈新. 火电厂锅炉干排渣技术的国产化 及应用 $[\mathrm{J}]$. 中国电力, 2007, 40(6): 20-23.

LIU Xiao, LIU Zhenqiang, CHEN Xin. Domestic production and application of dry slag disposal technology in coal-fired power plant[J]. Electric Power, 2007, 40(6): 20-23.

[3] 高继录, 冷杰, 许华, 等. $1000 \mathrm{MW}$ 机组干式排渣系统 对锅炉效率影响的试验研究 [J]. 热能动力工程, 2012, 27(5): 578-581, 595.

GAO Jilu, LENG Jie, XU Hua, et al. Experimental study of the influence of the dry type deslagging system of a $1000 \mathrm{MW}$ unit on the boiler efficiency[J]. Journal of Engineering for Thermal Energy and Power, 2012, 27(5): 578-581, 595.

[4] 范仁东. 从实测数据分析风冷干排渣系统对锅炉效率 的影响[J]. 电力技术, 2010, 19(7): 63-68.

FAN Rendong. Analysis of influence of air cooled dry-type slag removal system on boiler efficiency using practically measured data[J]. Electric Power Technology, 2010, 19(7): 63-68.

[5] 王建勋, 张艳辉, 谢鹏飞, 等. $W$ 型火焰锅炉配风方式 对 $\mathrm{NO}_{\mathrm{x}}$ 排放和经济性影响的试验研究[J]. 热能动力工 程, 2016, 31(1): 103-106, 143-144.

WANG Jianxun, ZHANG Yanhui, XIE Pengfei, et al. Experimental research of influence of air distribution mode for a W-flame boiler on $\mathrm{NO}_{\mathrm{x}}$ emission and boiler economy[J]. Journal of Engineering for Thermal Energy and Power, 2016, 31(1): 103-106, 143-144.

[6] 夏文静, 衡丽君, 何长征. 基于在线风量计算干式排渣 系统漏风量的试验研究[J]. 节能技术, 2015，33(192): 380-385.

XIA Wenjing, HENG Lijun, HE Changzheng. Experimental study on measuring the air leakage of dry-type slag removal system based on on-line air quantity[J]. Energy Conservation Technology, 2015, 33(192): 380-385.

[7] 许华, 张华伦, 王仕能, 等. 干、湿式除渣系统对锅炉 效率影响的研究[J]. 中国电力, 2013, 46(6): 1-4. XU Hua, ZHANG Hualun, WANG Shineng, et al. Study on the impact of dry and wet bottom ash handling systems on boiler efficiency[J]. Electric Power, 2013, 46(6): 1-4.

[8] 王雪彩, 孙树翁, 李明, 等. $600 \mathrm{MW}$ 墙式对冲锅炉低 氮燃烧技术改造的数值模拟 [J]. 中国电机工程学报, 2015, 35(7): 1689-1696.

WANG Xuecai, SUN Shuweng, LI Ming, et al. Numerical simulation on low $\mathrm{NO}_{\mathrm{x}}$ combustion technological transformation of a $600 \mathrm{MW}$ boiler with opposed wall swirling burners[J]. Proceedings of the CSEE, 2015, 35(7): 1689-1696.

[9] 李德波, 沈跃良, 徐齐胜, 等. 运用燃烧数值模拟分析 某台 660MW 超临界锅炉旋流燃烧器喷口烧损事故 [J]. 机械工程学报, 2013，49(16): 121-130.

LI Debo, SHEN Yueliang, XU Qisheng, et al. Numerical investigations on the key mechanisms of burnout of swirling combustors for $660 \mathrm{MW}$ supercritical unit swirl coal-fired combustion boiler[J]. Journal of Mechanical Engineering, 2013, 49(16): 121-130.

[10]李德波, 宋景慧, 徐齐胜, 等. 660MW 超超临界旋流 对冲燃煤锅炉 $\mathrm{NO}_{\mathrm{x}}$ 分布数值模拟 [J]. 动力工程学报, 2013，33(12)：913-919， 954.

LI Debo, SONG Jinghui, XU Qisheng, et al. Numerical simulation on $\mathrm{NO}_{\mathrm{x}}$ distribution in a $660 \mathrm{MW}$ ultra supercritical opposed firing boiler[J]. Journal of Chinese Society of Power Engineering，2013，49(16): 121-130.

[11] 李明, 王雪彩, 孙树翁, 等. 燃尽风射流形式对墙式对 冲煤粉锅炉低氮燃烧改造的影响 [J]. 动力工程学报, 2015，35(4): 263-269.

Li Ming, WANG Xuecai, SUN Shuweng, et al. Influence of overfire air jet form on low $\mathrm{NO}_{\mathrm{x}}$ retrofit effect of an opposed firing boiler[J]. Journal of Chinese Society of Power Engineering, 2015，35(4): 263-269.

[12] 刘亚明, 李方勇, 徐齐胜, 等. $600 \mathrm{MW}$ 对冲燃烧锅炉 $\mathrm{NO}_{\mathrm{x}}$ 排放特性的数值模拟 [J]. 动力工程学报, 2015, 35(5): 341-347.

LIU Yaming, LI Fangyong, XU Qisheng, et al. Numerical study on $\mathrm{NO}_{\mathrm{x}}$ emission characteristics of a $600 \mathrm{MW}$ opposed firing boiler[J]. Journal of Chinese Society of Power Engineering, 2015，35(5): 341-347.

[13] 李兵臣, 宋景慧, 沈跃良, 等. 二次风旋流强度可调范 围的数值模拟研究 [J]. 动力工程学报, 2012，32(12): 916-921.

LI Bingchen, SONG Jinghui, SHEN Yueliang, et al. Numerical study on adjustable range of secondary air swirl intensity [J]. Journal of Chinese Society of Power (下转第 226 页) 
in fluid filled piping systems[C]// ASME 2002 International Mechanical Engineering Congress and Exposition. American Society of Mechanical Engineers, 2002: 161-168.

[53] CHEER J, DALEY S. Broadband active control of noise and vibration in a fluid-filled pipeline using an array of non-intrusive structural actuators[C]// Inter-noise \& Noise-con Congress \& Conference, 2015.
作者简介：商夏，女，1992 年出生，博士研究生。主要研究方向为液压 系统振动与噪声控制。

E-mail: shangxia1007@163.com

周华(通信作者), 男, 1968 年出生, 博士, 教授, 博士研究生导师。主 要研究方向为水液压技术、电液控制工程。

E-mail: hzhou@sfp.zju.edu.cn

杨华勇, 男, 1961 年出生, 博士, 教授, 博士研究生导师, 中国工程院 院士。主要研究方向为电液控制元件与系统、流体动力与机电系统的 节能。

E-mail: yhy@zju.edu.cn

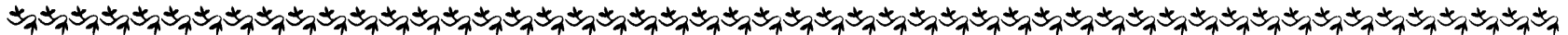

(上接第 215 页)

Engineering, 2012, 32(12): 916-921.

[14] 董康, 周吴, 杨玉, 等. 二次风量对旋流燃烧器气固流 动特性的影响 [J]. 浙江大学学报, 2014, 48(12): 2162-2171.

DONG Kang, ZHOU Hao, YANG Yu, et al. Influence of mass flow rate of secondary air on gas/solid characteristics of a swirl burner[J]. Journal of Zhejiang
University, 2014, 48(12): 2162-2171.

作者简介: 刘铭媛(通信作者), 女, 1983 年出生, 工程师。主要研究 方向为燃煤发电机组清洁高效燃烧技术和污染物控制技术，煤粉燃烧 数值模拟。

E-mail: mingyuan716@163.com

陈国庆, 男, 1982 年出生, 高级工程师。主要研究方向燃煤发电机组清 洁高效燃烧技术和污染物控制技术。

E-mail: chengqhit@163.com 